\title{
How can natural products serve as a viable source of lead compounds for the development of new/novel anti-malarials?
}

\author{
Eric Guantai ${ }^{1}$, Kelly Chibale ${ }^{1,2^{*}}$
}

\begin{abstract}
Malaria continues to be an enormous global health challenge, with millions of new infections and deaths reported annually. This is partly due to the development of resistance by the malaria parasite to the majority of established anti-malarial drugs, a situation that continues to hamper attempts at controlling the disease. This has spurred intensive drug discovery endeavours geared towards identifying novel, highly active anti-malarial drugs, and the identification of quality leads from natural sources would greatly augment these efforts. The current reality is that other than compounds that have their foundation in historic natural products, there are no other compounds in drug discovery as part of lead optimization projects and preclinical development or further that have originated from a natural product start-point in recent years. This paper briefly presents both classical as well as some more modern, but underutilized, approaches that have been applied outside the field of malaria, and which could be considered in enhancing the potential of natural products to provide or inspire the development of anti-malarial lead compounds.
\end{abstract}

\section{Background}

Malaria remains the most devastating tropical disease, with staggering infection and mortality statistics. The WHO World Malaria Report 2008 estimated that there were approximately 247 million malaria cases among 3.3 billion people at risk in 109 countries where malaria is currently considered prevalent; $87 \%$ of these cases were reported in the African region. The disease caused nearly one million deaths, $91 \%$ of which were in Africa, and $85 \%$ of these were of children under 5 years [1]. The main challenge to the effective management of diagnosed malaria cases has been, and continues to be, the resistance of the causative microorganisms to known anti-malarials, which results in the non-resolution of symptoms, recrudescence, and ultimately in treatment failure [2-4].

Various strategies have been embraced in the fight against malaria in general, and anti-malarial drug resistance in particular, and include the improvement of prescribing habits and therapeutic protocols, prevention of

\footnotetext{
* Correspondence: Kelly.Chibale@uct.ac.za

${ }^{1}$ Department of Chemistry, University of Cape Town, Rondebosch, 7701,

South Africa

Full list of author information is available at the end of the article
}

infection and the use of combination therapies, with particular emphasis being placed on artemisinin-based combination therapy (ACT) [2,3,5-7]. In tandem with these efforts has been the intensive drug discovery effort aimed at developing new anti-malarial drugs or modifying existing ones, and which targets the identification of novel compounds that exhibit excellent experimental and clinical anti-malarial efficacy without showing any evidence of resistance. Unfortunately the current reality is that other than compounds that have their foundation in historic natural products (such as quinine, artemisinin, hydroxynaphthoquinones, doxycyclin, clindamycin, and azithromycin), there are no other compounds in preclinical development or further that have originated from a natural product start-point in recent years. There are not even any compounds in current antimalarial lead optimization projects that have come from natural products in recent years. Many natural products have shown potent anti-plasmodial effects but, for a variety of reasons, including chemical tractability issues, these have not been pushed forward into hit-to-lead drug discovery projects.
C Biomed Central

(ㄷ) 2011 Guantai and Chibale; licensee BioMed Central Ltd. This is an open access article distributed under the terms of the Creative Commons Attribution License (http://creativecommons.org/licenses/by/2.0), which permits unrestricted use, distribution, and reproduction in any medium, provided the original work is properly cited. 


\section{Generic approaches to anti-malarial natural product drug discovery}

Nature is, as ever, an extremely rich source of potential anti-malarial agents [8], with the anti-malarial drugs quinine and artemisinin being outstanding examples of therapeutic natural products $[9,10]$.

Following the serendipitous or rational discovery of a biologically active natural material, the conventional approach to natural product development has been the bioassay-guided fractionation of extracts derived from such material, and the subsequent isolation and characterization of pure, active compounds. With artemisinin as an illustrative example (Figure 1) [9-12], the identification of promising compounds in this way usually triggers medicinal chemistry efforts geared towards the total synthesis of the identified compounds and/or the generation of analogs, and which are aimed at providing a supplementary source of the product for further study, revealing structure-activity relationships (SAR), identifying more potent analogs and/or overcoming challenging physicochemical and biological properties. Using comparable approaches, the continuing search for new anti-malarial drugs from natural sources has led to the identification of an impressive range of structurally diverse compounds from a variety of chemical classes [9,13-15].

\section{Challenges facing conventional natural product drug discovery}

The challenges facing conventional anti-malarial natural product drug discovery (and indeed natural product drug discovery in general) are many, and range from the basic problems of reliable access and supply, seasonal or environmental variations in the composition of living organisms and loss of source through extinction or legislation, to the more practical concerns associated with the complexity of the mixtures after fractionation, the isolation of very small quantities of bioactive substance and challenging physicochemical properties such as solubility and stability [16].

Part of the solution may be either to look for ways to supplement the natural sources to meet the existing demand of known bioactive molecules, or to consciously widen the scope of the biodiversity that is sampled for natural product drug discovery by, for example, the exploration of extreme habitats not routinely considered $[17,18]$. Recent advances in biotechnology and the understanding of the genomics of natural product biosynthesis may enable the efficient production of the same bioactive molecules from plant-cell cultures and genetically engineered microorganisms [19], such as the large-scale microbial production of the artemisinin precursors amorpha-4,11-diene and artemisinic acid from genetically modified Saccharomyces cerevisiae and
Escherichia coli[20]. Sources that are known to be rich in secondary molecules active against other diseases could also be targeted as potential sources of anti-malarial compounds. For example, the study of marine actinomycetes, long recognized as a rich source of secondary metabolites with anticancer activity, yielded the highly potent anti-malarial compound salinosporamide A (Figure 1), isolated from the marine actinomycete Salinispora tropica[21].

However, it is noteworthy that most anti-malarial compounds isolated from natural sources are usually only moderately active, or possess challenging physicochemical and biological properties, and as such represent 'hits' rather than actual lead drug candidates. It, therefore, becomes apparent that considerable effort needs to be made to complement conventional natural product drug discovery and boost the chances of the successful identification of quality lead compounds from natural products, including their development from naturally derived hits. Such efforts must include the incorporation of what may be considered as more modern drug discovery strategies into anti-malarial natural product drug discovery endeavors (Figure 2). This would arguably increase the chances of successful identification and/or design of new potential leads, as well as considerably enhance the range and depth of knowledge and information that is derived from these discovery efforts.

With past and recent examples, the potential of some of these approaches is highlighted.

\section{Drug combinations}

As mentioned earlier, one of the strategies for evading the development of resistance to anti-malarials is the use of combination therapies, which basically involves the co-administration of two or more anti-malarial agents. Drugs selected for combination in this way usually have a demonstrated synergistic effect when applied together, thereby allowing for the use of lower doses and achievement of more rapid therapeutic outcomes while taking advantage of the lower risk of the concurrent development of resistance by the microorganism to the co-administered agents. Although the emphasis is currently on artemisinin-based combination therapy (ACT) [5-7], the identification of new and efficacious combinations remains a priority, and the identification of new, active, naturally-derived compounds could provide additional possibilities. The potential of combinations of established anti-malarial drugs with other bioactive compounds derived from natural sources has already been demonstrated experimentally. For example, the combination of chloroquine and a mixture of febrifugine and isofebrifugine (isolated from the leaves of Hydrangea macrophylla) was found to be more 


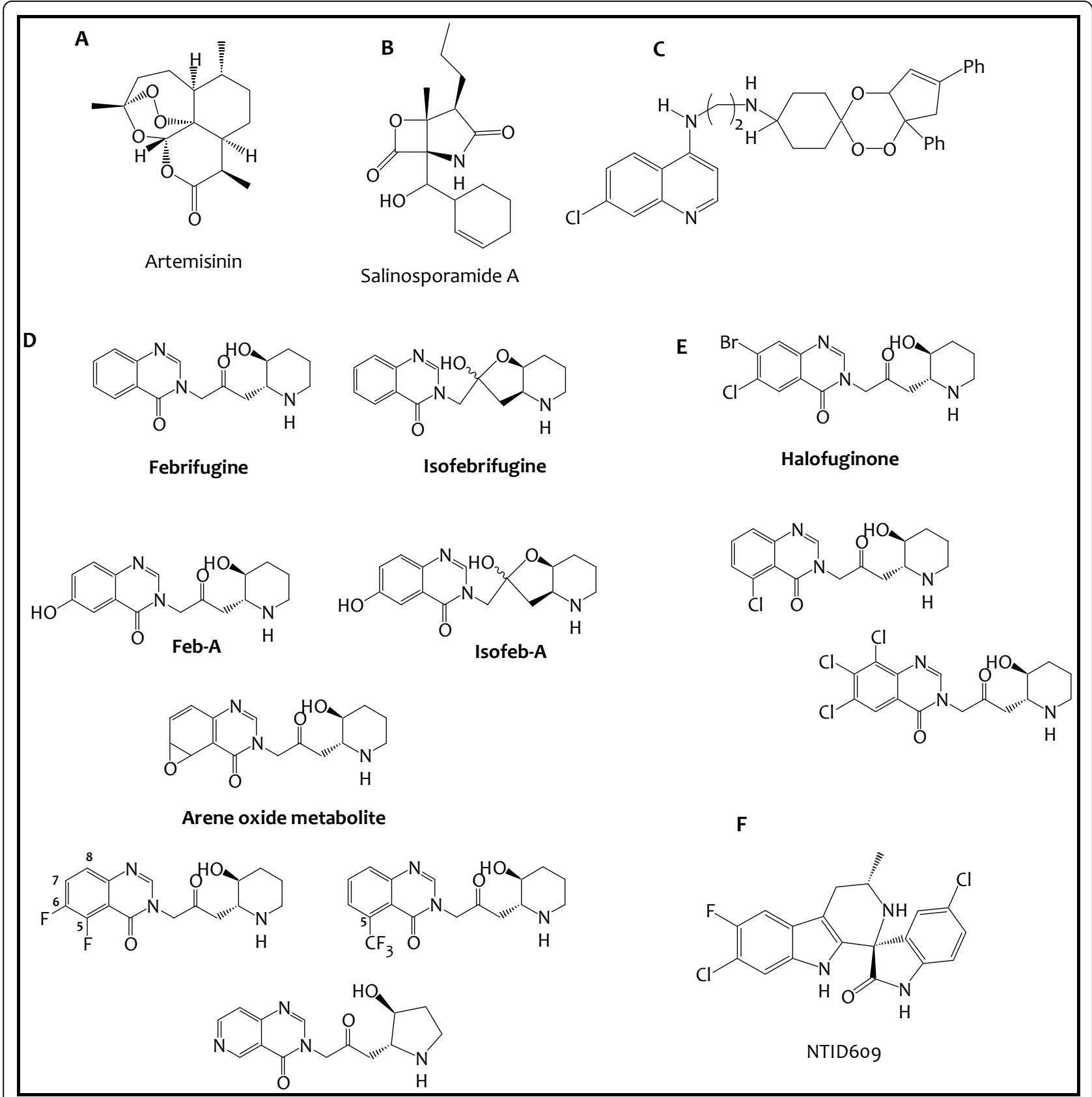

Figure 1 A: structure of artemisinin; B: salinosporamide A, an antimalarial compound isolated from marine actinomycetes; C: an example of a highly active trioxaquine; $\mathbf{D}$ : febrifugine, isofebrifugine, metabolite feb-A and its synthetic analog isofeb-A, the arene oxide metabolite and examples of potent but less hepatotoxic halogenated febrifugine analogs; $\mathbf{E}$ : Halofuginone, as well as some highly active febrifugine analogs identified by virtual screening; F: NTID609, an extremely active and highly promising anti-malarial spiroindolone.

active in vivo than the individual components of the combination [22].

\section{Dual drugs}

Dual drugs, also known as hybrid compounds, refer to single chemical entities that consist of two drugs/active compounds/pharmacophoric units linked together covalently by a linker [23]. This aims to take advantage of the observed (or anticipated) synergistic or additive pharmacological activities of the hybrid components and enable the identification of highly active novel chemical entities. Highly active trioxaquines have been developed by covalently linking a 1,2,4-trioxane motif (a peroxide entity derived from the highly active natural sesquiterpene artemisinin) to a 4-aminoquinoline moiety (borrowed from chloroquine and other chloroquinoline- 


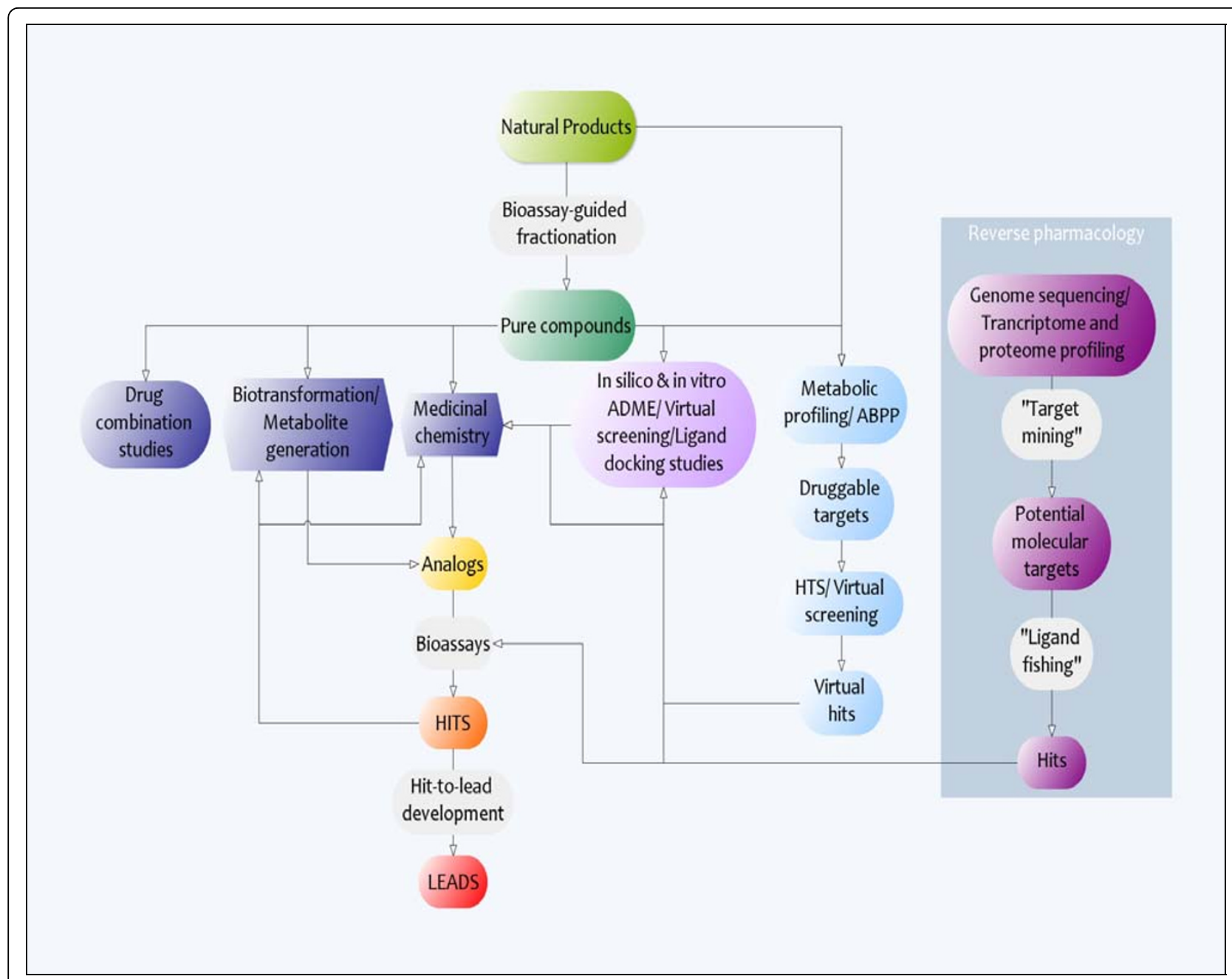

Figure 2 Development of leads from natural products.

based anti-malarials) via an appropriate spacer. Several of these trioxaquine derivatives (Figure 1) were found to be extremely potent anti-plasmodial agents [24].

\section{Metabolism and metabolite identification studies}

In silico (computational) and in vitro metabolism studies are primarily aimed at assessing the metabolic stability of promising hit and lead compounds, and are usually geared at providing useful information to guide and/or help in the interpretation of the results from subsequent pharmacokinetic studies and in vivo assays. In addition, such information also enables the drug discovery scientists to design and synthesize analogs of the primary compound(s) that are potentially more stable to metabolism and are likely to have improved oral bioavailability profiles [25].

However, and probably just as importantly, the in vitro generation and identification of primary metabolites of a compound can be an effective way of generating chemical diversity and augmenting the efforts aimed at identifying novel active compounds based on an identified hit. Pharmacologically active metabolites have been found to contribute significantly to the overall observed in vivo anti-malarial activity of many compounds. For example, $\mathrm{N}$-desethylchloroquine, the primary metabolite of chloroquine, exhibits potent anti-malarial activity comparable to that of chloroquine [26,27]. This concept of pharmacologically active metabolites is just as applicable to anti-malarial hits derived from natural sources. Oshima and co-workers [28] reported the isolation and structural elucidation of two primary metabolites of febrifugine after its incubation for 1 hour at $37^{\circ} \mathrm{C}$ with mouse liver S9. Febrifugine is a highly potent antimalarial alkaloid isolated from the roots, stem and leaves of the plant Dichroa febrifuga, a Chinese herb locally referred to as Chang Shan[29]. One of the major metabolites designated feb-A (Figure 1) showed extremely potent in vitro anti-plasmodial activity. Isofeb-A 
(Figure 1), a synthetic analog of feb-A, was even more potent than febrifugine in vitro, and had a significantly higher selectivity index [28].

In addition, metabolism studies may be useful in understanding the in vivo toxicity of a particular compound; this is because any toxic effects observed after the administration of a particular compound could be due to the generation of toxic metabolites. Such information derived from metabolism studies would then be crucial in the design of potent compounds devoid of the unwanted toxicity of the parent compound. Data from SAR, toxicity and metabolism studies of febrifugine was used to design a series of febrifugine analogs with the aim of identifying compounds with attenuated hepatotoxicity. This toxicity has precluded the development of febrifugine as a potential clinical drug, and is mediated by its arene oxide metabolite (Figure 1). Several analogs were identified that retained potent anti-plasmodial activity in vitro but were considerably less cytotoxic than febrifugine based on an in vitro cytotoxicity assay on rat hepatocytes. [30,31].

\section{Molecular modeling and docking tools}

The use of modern molecular modeling and docking tools may be applied to enrich the process of derivatization of natural products by guiding or complementing the rational design and selection of more promising derivatives for subsequent synthesis and biological evaluation. An example of such a tool has been developed and validated for the analysis of artemisinin-based analogs as potential haem inhibitors [32].

\section{Natural product-derived pharmacophores and template-based virtual screening}

Upon the elucidation of the structure of a novel antimalarial compound from natural sources, and possibly informed by preliminary SAR data, it may be possible to identify its basic pharmacophoric unit(s). This not only facilitates more elaborate SAR studies, but also allows for the systematic searches of databases and libraries of known compounds (from both natural and synthetic sources) for structural analogs. Analogs identified in this way can then be acquired/ synthesized and assayed alongside the primary compound, and thereby provide additional SAR information and possibly even lead to the identification of additional active compounds that would have otherwise remained unidentified. The piperidinyl(acetonyl)quinazoline moiety, a structural unit derived from febrifugine, was used as a template in the search for analogs in the Walter Reed Army Institute of Research (WRAIR) Chemical Information System (CIS) database. In this way, potent analogs of febrifugine were identified that also had significantly reduced in vitro cytotoxicity against a variety of mammalian cell lines [33].

\section{Natural product databases}

An impressively large number of novel naturally-derived compounds have been isolated and characterized over the years. The creation of virtual and/or physical repositories of these compounds would help to keep track of these compounds and simultaneously create a rich resource that can be tapped for drug discovery efforts. Examples of such databases already exist [34,35], and these can be valuable for fragment- or ligand-based virtual screening, as well as for ligand docking studies on a variety of known protein targets. Physical repositories of crude and purified natural products can provide compound libraries for HTS, metabolic profiling, reverse pharmacology and related endeavors.

A recent example of the successful application of such a library of natural products and natural product-like molecules is in the discovery of highly active anti-malarial spiroindolone compounds. The identification of this class of compounds began with the screening of about 12,000 pure natural products and synthetic compounds with structural features found in natural products. Hits from this initial screen were then filtered based on in vitro anti-plasmodial activity, cytotoxicity against mammalian cells, physicochemical and pharmacokinetic properties. A compound related to the spiroazepineindole class was thereby identified as a starting point for medicinal chemistry efforts that eventually led to the identification of the highly active compound NITD609 (Figure 1). This compound was found to be as effective as artesunate, showed no evidence of diminished potency against drug-resistant strains, exhibited favorable in vitro solubility and permeability and did not show cytotoxicity across several human cell lines [36,37].

Physical repositories of natural products, such as the one set up by the Defense Science and Technology Organization in Australia, also offer a resource for antimalarial screening. Screening of some of the extracts from this library revealed that some extracts, particularly those from the Grevillea species, possessed quite strong anti-plasmodial activity [38].

\section{Target-identification and reverse pharmacology}

In addition to serving as a source of anti-malarial lead compounds, natural products can also add value to other techniques available for anti-malarial drug discovery. This is particularly with respect to the identification of new anti-malarial drug targets.

Metabolic profiling offers an exciting approach to the identification of (oftentimes new) molecular targets of biologically active molecules, and involves the use of tools such as NMR $[39,40]$ and Mass spectroscopy $[41,42]$ in the assessment of the metabolic response of an organism/cell/parasite following exposure to a bioactive molecule [43]. Bioactive natural products, by virtue 
of their chemical diversity, are particularly suited to act as probes for the identification of new molecular targets in this way $[43,44]$. The metabolic profiling of the malaria parasite has been reported $[39,40,45]$, which would suggest the latent potential of this approach.

Another approach by which natural products may be useful in the identification of druggable targets is Activity-Based Protein Profiling (ABPP) [46]. Proteinreactive natural products (or privileged structures derived from them) can be useful in the development of ABPP probes [47]. For example, compound E-64, a cysteine protease inhibitor isolated from cultures of Aspergillus japonicus [48], is known to interact with plasmodial cysteine proteases [49] and has been successfully applied as a scaffold for the development of ABPP probes [50]. Proteins/enzymes identified in this way can then be investigated further as potential therapeutic targets by both classical mechanism of action studies or reverse pharmacology [51].

Reverse pharmacology offers an alternative means by which the molecular targets of biologically active compounds may be identified. Rather than apply a bioactive molecule in the identification of a molecular target, reverse pharmacology begins with the initial identification of potential protein targets (such as enzymes and receptors) by the application of bioinformatics tools that exploit the vast DNA-sequence databases provided by intensive genomic research. The potential targets identified in this way are then cloned and used to screen candidate ligands, which may include natural products [52]. As far as antimalarial drug discovery is concerned, this approach has been rendered quite feasible by the successful sequencing of the genome of the human malaria parasite P. falciparum [53], now available as a database for vaccine development and drug discovery applications [54].

It has been argued that libraries of natural products and their derivatives are actually better suited for such screens simply because they span a much broader chemical space than most synthetic compound libraries currently available for this purpose [55]. It has even been proposed that screening libraries should be intentionally optimized by adding to them molecules with biogenic or natural-product-like scaffolds [56], further underlining the value of natural products to such strategies.

\section{Conclusion}

Natural products may be lead compounds in themselves, or more likely may serve as hits that may be useful in providing pharmacophores/templates that can guide the design of potentially superior analogs and/or the mining of existing databases of synthetic and semi-synthetic compounds for previously untested and potentially active analogs. Promising analogs designed or identified in this way may be expanded and developed further by introduction of (bio)isosteric substitutions suggested by scaffold hopping techniques, or by the application of in silico (computational) and in vitro profiling of physicochemical and $\operatorname{ADME}(\mathrm{T})$ parameters to enable the rational design of potentially improved/superior analogs. The in vitro generation of metabolites can ably complement synthetic and semi-synthetic efforts at derivatization of primary, naturally derived, bioactive molecules, thereby broadening the chemical diversity around these molecules and increasing the likelihood of identifying promising compounds. Natural products can also find wide application in target-identification studies - metabolic profiling of the malaria parasite has been reported and the possible role of natural products acknowledged; ABPP probes based on scaffolds known to interact with malarial proteins have been developed; the elucidation and reporting of the malaria genome affords the opportunity for reverse pharmacology.

The potential of natural products to provide or inspire the development of anti-malarial lead compounds is, therefore, really quite evident. However, to raise the chances of the actual realization of this potential, it has become necessary to think beyond the confines of conventional natural-product drug discovery. The application of a wide variety of scientific tools and the close and interactive collaboration of experts in diverse scientific disciplines (such as chemistry, pharmacology, molecular biology and genetics) has become practically obligatory if these truly multi-disciplinary efforts are to indeed be successful. The fact that literature on the application of some of these approaches towards antimalarial drug discovery based on natural products is sparse is indicative of their underutilization in this regard, a situation that should arguably be addressed.

\section{Acknowledgements}

This article has been published as part of Malaria Journal Volume 10 Supplement 1, 2011: Natural products for the control of malaria. The full contents of the supplement are available online at http://www. malariajournal.com/supplements/10/S1.

\section{Author details}

'Department of Chemistry, University of Cape Town, Rondebosch, 7701, South Africa. ${ }^{2}$ Institute of Infectious Disease and Molecular Medicine, University of Cape Town, Rondebosch, 7701, South Africa.

\section{Competing interests}

The authors declare that they have no competing interests.

Published: 15 March 2011

\section{References}

1. World Health Organization: World Malaria Report 2008.

2. World Health Organization: Guidelines for the treatment of malaria. 2006.

3. Price RN, Nosten F: Drug resistant falciparum malaria: clinical consequences and strategies for prevention. Drug Resist Update 2001, 4:187-196 
4. World Health Organization Expert Committee on Malaria: WHO Technical Report Series 2000.

5. White $\mathrm{N}$ : Antimalarial drug resistance and combination chemotherapy. Phil Trans R Soc Lond B biol Sci 1999, 354:739-749.

6. Mutabingwa TK: Artemisinin-based combination therapies (ACTs): best hope for malaria but inaccessible to the needy. Acta Trop 2005, 95:305-315.

7. Edwards G, Biagini GA: Resisting resistance: dealing with the irrepressible problem of malaria. BriJ Clin Pharmacol 2006, 61:690-693.

8. Itokawa H, Morris-Natschke SL, Akiyama T, Lee K-H: Plant-derived natural product research aimed at new drug discovery. J Nat Med 2008, 62:263-280.

9. Saxena S, Pant N, Jain DC, Bhakuni RS: Antimalarial agents from plant sources. Curr Sci 2003, 85:1314-1329.

10. Wang M-W, Hao X, Chen K: Biological screening of natural products and drug innovation in China. Phil Trans R Soc B 2007, 362:1093-1105.

11. Klayman DL: Quinghaosu (artemisinin): An antimalarial drug from China. Science 1985, 228:1049-1055.

12. Haynes RK: From artemisinin to new artemisinin antimalarials: biosynthesis, extraction, old and new derivatives, stereochemistry and medicinal chemistry requirements. Curr Top Med Chem 2006, 6:509-537.

13. Kaur K, Jain M, Kaur T, Jain R: Antimalarials from nature. Bioorg Med Chem 2009, 17:3229-3256.

14. Gademann K, Kobylinska J: Antimalarial natural products of marine and freshwater origin. Chem Rec 2009, 9:187-198.

15. Batista R, Silva AJ Jnr, Oliviera AB: Plant-derived antimalarial agents: new leads and efficient phytomedicines. Part II. Non-alkaloidal natural products. Molecules 2009, 14:3037-3072.

16. Li JW-H, Vederas JC: Drug discovery and natural products: end of an era or an endless frontier? Science 2009, 325:161-165.

17. Pettit RK: Culturability and secondary metabolite diversity of extreme microbes: expanding contribution of deep sea and deep-sea vent microbes to natural product discovery. Mar Biotechnol 2010, Online publication:DOI 10.1007/s10126-010-9294-y.

18. Harvey A: Strategies for discovering drugs from previously unexplored natural products. Drug Disc Today 2000, 5:294-300.

19. John JE: Natural products as lead-structures: a role for biotechnology (Editorial). Drug Discov Today 2010, 15:409-410.

20. Zeng $Q$, Qui F, Yuan L: Production of artemisinin by genetically-modified microbes. Biotechnol Lett 2008, 30:581-592.

21. Prudhomme J, McDaniel E, Ponts N, Bertani S, Fenical W, Jensen P, le Roch K: Marine Actinomycetes: a new source of compounds against the human malaria parasite. PLOS ONE 2008, 3:e2335.

22. Ishih A, Suzuki T, Watanabe M, Miyase T, Terada M: Combination effects of chloroquine with the febrifugine and isofebrifugine mixture against a blood-induced infection with chloroquine-resistant Plasmodium berghei NK65 in ICR Mice. Phytother Res 2003, 17:1234-1236.

23. Walsh JJ, Bell A: Hybrid drugs for malaria. Curr Pharm Des 2009, 15:2970-2985

24. Dechy-Cabaret D, Benoit-Vical F, Robert A, Meunier B: Preparation and antimalarial activities of "Trioxaquines", new modular molecules with a trioxaneskeleton linked to a 4-aminoquinoline. Chembiobhem 2000, 1:281-283

25. Kerns EK, Di L: Drug-like properties: concepts, structure, design and methods: from ADME to toxicity optimization. Academic Press/Elselvier 2008.

26. Projean D, Baune B, Farinotti R, Flinois JP, Beaune P, Taburet AM, Ducharme $A$ : In vitro metabolism of chloroquine: identification of CYP2C8, CYP3A4 and CYP2D6 as the main isoforms catalyzing Ndesethylchloroquine formation. Drug Metab Dispos 2003, 31:748-754.

27. Fu S, Björkman A, Wåhlin B, Ofori-Adjei D, Ericsson O, Sjöqvist F: In vitro activity of chloroquine, the two enantiomers of chloroquine, desethylchloroquine and pyronaridine against Plasmodium falciparum. Br J Clin Pharmacol 1986, 22:93-96.

28. Hirai S, Kikuchi H, Kim H-S, Begum K, Wataya Y, Tasaka H, Miyazawa Y, Yamamoto K, Oshima Y: Metabolites of febrifugine and its synthetic analogue by mouse liver $\mathrm{S} 9$ and their antimalarial activity against Plasmodium malaria parasite. J Med Chem 2003, 46:4351-4359.

29. Kuehl FA, Spencer CF, Folkers K: Alkaloids of Dichroa febrifuga Lour. J Am Chem Soc 1948, 70:2091-2093.
30. Zhu S, Meng L, Zhang Q, Wei L: Synthesis and evaluation of febrifugine analogues as potential antimalarial agents. Bioorg Med Chem Lett 2006, 16:1854-1858.

31. Zhu S, Zhang Q, Gudise C, Wei L, Smith E, Zeng Y: Synthesis and biological evaluation of febrifugine analogues as potential antimalarial agents. Bioorg Med Chem 2009, 17:4496-4502.

32. Srivastava M, Singh H, Naik PK: Molecular modeling evaluation of the antimalarial activity of artemisinin analogues: molecular docking and rescoring using Prime/MM-GBSA Approach. Curr Res J Biol Sci 2010, 2:83-102.

33. Jiang $S$, Zeng $Q$, Gettayacamin $M$, Tungtaeng A, Wannaying S, Lim A, Hansukjariya P, Okunji CO, Zhu S, Fang D: Antimalarial activities and therapeutic properties of febrifugine analogs. Antimicrob Agents Chemother 2005, 49:1169-1176.

34. Dictionary of natural products. 2010.

35. Lei J, Zhou J: A marine natural product database. J Chem Inf Comput Sci $2002,42: 742-748$.

36. Yeung BKS, Zou B, Rottmann M, Lakshminarayana SB, Ang SH, Leong SY, Tan J, Wong J, Keller-Maerki S, Fischli C, Goh A, Schmitt EK, Krastel P, Francotte E, Kuhen K, Plouffe D, Henson K, Wagner T, Winzeler EA, Petersen F, Brun R, Dartois V, Diagana TT, Keller TH: Spirotetrahydro bCarbolines (Spiroindolones): A New Class of Potent and Orally Efficacious Compounds for the Treatment of Malaria. J Med Chem 2010, 53:5155-5164.

37. Rottmann M, McNamara C, Yeung BKS, Lee MCS, Zou B, Russell B, Seitz P, Plouffe DM, Dharia NV, Tan J, Cohen SB, Spencer KR, González-Páez GE, Lakshminarayana SB, Goh A, Suwanarusk R, Jegla T, Schmitt EK, Beck HP, Brun R, Nosten F, Renia L, Dartois V, Keller TH, Fidock DA, Winzeler EA, Diagana TT: Spiroindolones, a potent compound class for the treatment of malaria. Science 2010, 329:1175-1180.

38. Ovenden SPB, Chavchich M, Cobbe M, Pigott EJ, Laws MJ, Edstein MD: Preparation of a natural product extract library for investigation against disease states specific to defence health: a mini long range research project. Defence Science and Technology Organisation, Department of Defence, Australian Government 2009, DSTO-TR-2255.

39. Teng R, Junankar PR, Bubb WA, Rae C, Mercier P, Kirk K: Metabolite profiling of the intraerythrocytic malaria parasite Plasmodium falciparum by 1H NMR spectroscopy. NMR Biomed 2009, 22:292-302.

40. Lian L-Y, Al-Helal M, Roslaini AM, Fisher N, Bray PG, Ward SA, Biagini GA: Glycerol: An unexpected major metabolite of energy metabolism by the human malaria parasite. Malar J 2009, 8, doi:10.1186/1475-2875-8-38.

41. Cheng K-W, Wong C-C, Wang M, He Q-Y, Chen F: Identification and characterization of molecular targets of natural products by mass spectrometry. Mass Spectrom Rev 2010, 29:126-155.

42. Holmes E: The evolution of metabolic profiling in parasitology. Parasitology 2010, 137:1437-1449.

43. Pucheault M: Natural products: chemical instruments to apprehend biological symphony. Org Biomol Chem 2008, 6:424-432.

44. Harrigan GG, Brackett DJ, Boros LG: Medicinal chemistry, metabolic profiling and drug target discovery: a role for metabolic profiling in reverse pharmacology and chemical genetics. Mini-Rev Med Chem 2005, 5:13-20.

45. Cassera MB, Merino EF, Peres VJ, Kimura EA, Wunderlich G, Katzin AM: Effect of fosmidomycin on metabolic and transcript profiles of the methylerythritol phosphate pathway in Plasmodium falciparum. Mem Inst Oswaldo Cruz 2007, 102:377.

46. Harvey AL: Natural products in drug discovery. Drug Disc Today 2008, 13:894-901.

47. Cravatt BF, Wright AT, Kozarich JW: Activity-based protein profiling: from enzyme chemistry to proteomic chemistry. Annu Rev Biochem 2008, 77:383-414.

48. Barrett AJ, Kembhavi AA, Brown MA, Kirschke H, Knight CG, Tamai M, Hanada K: L-trans-Epoxysuccinyl-leucylamido(4-guanidino)butane (E-64) and its analogues as inhibitors of cysteine proteinases including cathepsins B, H and L. Biochem J 1982, 201:189-198.

49. Rosenthal PJ: Cysteine proteases of malaria parasites. Int J Epidemiol 2004, 34:1489-1499.

50. Greenbaum D, Medzihradszky KF, Burlingame A, Bogyo M: Epoxide electrophiles as activity-dependent cysteine protease profiling and discovery tools. Chem Biol 2000, 7:569-581. 
51. Böttcher T, Pitscheider M, Sieber SA: Natural products and their biological targets: proteomic and metabolomic labeling strategies. Angew Chem Int Ed Eng/ 2010, 49:2680-2698.

52. Takenaka T: Classical vs reverse pharmacology in drug discovery. BJU Int 2001, 88 Suppl 2:7-10.

53. Gardner MJ, Hall N, Fung E, White O, Berriman M, Hyman RW, Carlton JM, Pain A, Nelson KE, Bowman S, et al: Genome sequence of the human malaria parasite Plasmodium falciparum. Nature 2002, 419:498-511.

54. The Plasmodium Genome Database Collaborative: PlasmoDB: An integrative database of the Plasmodium falciparum genome. Tools for accessing and analyzing finished and unfinished sequence data. Nucleic Acids Res 2001, 29:66-69.

55. Bauer RA, Wurst JM, Tan DS: Expanding the range of 'druggable' targets with natural product-based libraries: an academic perspective. Curr Opin Chem Biol 2010, 14:308-314.

56. Hert J, Irwin JJ, Laggner C, Keiser MJ, Shoichet BK: Quantifying Biogenic Bias in Screening Libraries. Nat Chem Biol 2009, 5:479-483.

doi:10.1186/1475-2875-10-S1-S2

Cite this article as: Guantai and Chibale: How can natural products serve as a viable source of lead compounds for the development of new/ novel anti-malarials? Malaria Journal 2011 10(Suppl 1):S2.

\section{Submit your next manuscript to BioMed Central} and take full advantage of:

- Convenient online submission

- Thorough peer review

- No space constraints or color figure charges

- Immediate publication on acceptance

- Inclusion in PubMed, CAS, Scopus and Google Scholar

- Research which is freely available for redistribution

Submit your manuscript at www.biomedcentral.com/submit 